\title{
International survey of ophthalmic anaesthesia service provision, protection of anaesthesia providers and patients during COVID-19 pandemic: a wake-up call
}

\author{
Shashi B. Vohra $\mathbb{D}^{1 凶}$ and Chandra M. Kumar $\mathbb{D}^{2,3}$ \\ ๑) Crown 2022, corrected publication 2022
}

\begin{abstract}
AIMS: This international survey was conducted to study the impact of Covid-19 pandemic on the provision and practices of ophthalmic anaesthesia, evaluate the methods employed by parent ophthalmic units for safeguarding their anaesthesia providers and patients during lockdown, and to assess pandemic's effect on anaesthesia providers as individuals. The study was done with the hope that the results will help in protecting patients and safeguarding precious human resource by better management if this pandemic was to continue or there was to be another pandemic.

METHODS: An anonymous questionnaire survey was distributed electronically between December 2020-January 2021 to the practicing ophthalmic anaesthesia providers in different parts of the world.

RESULTS: The survey identified that apart from reducing elective operating services, the ophthalmic units were ill prepared for the pandemic and the overall management was lacklustre. There was a definite lack of effective peri-operative patient screening, and, streaming processes. Measures for personal protection of staff were not optimal especially during regional/local ophthalmic anaesthesia. Severity of the pandemic, sudden job plan changes, and redeployment to intensive care units/acute covid wards had an adverse psychological impact on the affected staff.

CONCLUSION: Ophthalmic anaesthesia services worldwide have had poor attentiveness to the life-threatening menace and reality of Covid-19 pandemic. A review of the institutional practices to address correctible deficiencies is urgently required. Robust, mandatory, elective, timely preventative strategies need to be implemented to protect patients, and, the precious ophthalmic workforce from potential adverse physical and psychological injuries.
\end{abstract}

Eye (2023) 37:548-553; https://doi.org/10.1038/s41433-022-01979-7

\section{INTRODUCTION}

Covid-19 pandemic has affected every corner of the planet, disturbed every human life in one form or another. The invisible airborne novel virus (SARS-CoV-2) with no pre-existing immunity in humans has cost millions of lives of various ages. It is truly lamentable that Dr Li Wenliang, an ophthalmologist, who was amongst the first few to raise the alarm about virulence and novelty of SARS-CoV-2, had his warnings ignored by relevant authorities [1]. He sadly died after contracting this deadly virus soon after its discovery in Wuhan, China.

Ophthalmic local/regional anaesthesia (LA/RA) is provided by both ophthalmologists and anaesthetists, the latter also provide sedation and general anaesthesia to patients spanning a wide spectrum of ages. It is now well established that the wildfire like human-to-human spread of this virus occurs primarily by inhalation of contagion laden aerosol as well as direct contact with oral, nasal or even ocular route via contaminated hands. While it is well recognised that general anaesthesia in a Covid-19 positive patient creates a high ambient viral load exposing anaesthesia providers to an extremely high risk, what is not acknowledged is that LA/RA for eye may also pose similar dangers. This risk of droplet/ aerosol transmission may be perhaps considered even higher during ophthalmic LA/RA compared to other peripheral regional nerve blocks as anaesthesia provider's face is literally inches away from the patient's airway.

Respiratory syndrome-coronavirus (SARS-CoV), with its high homology with the SARS-CoV-2 genome, has been well known to be transmitted by inoculation of conjunctiva, nasal, and oral mucosa. SARS-CoV had been shown to be present in the tears of three patients with early onset (within 9 days) by RT-PCR [2-4]. Conjunctivitis is present as the first sign In $0.8-3 \%$ of patients infected with Covid-19 [5]. Conjunctival hyperaemia, chemosis, retinal micro and overt flame-shaped haemorrhages, cotton wool spots, dilated veins, and tortuous vessels have all been described [6]. Ocular tissues serve as a reservoir for the SARS-CoV-2 virus from where it can migrate down to patient's respiratory tract [7], becoming a source of onwards transmission.

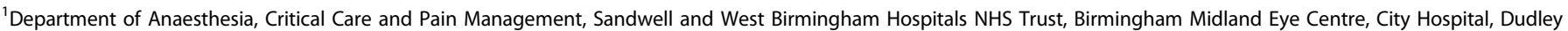

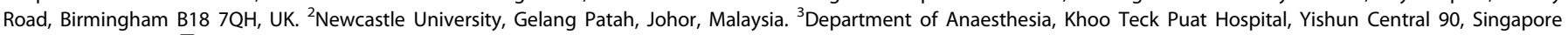
768828, Singapore. ${ }^{凶}$ email: shashi@vohra.org.uk
} 


\section{Aims}

We conducted this international survey with the primary aim to gauge the effect of pandemic on provision and practice of the ophthalmic anaesthesia and study the methods employed by parent ophthalmic units for protection of patients, and safeguarding health of their ophthalmic anaesthesia providers. The secondary aim was to assess the individual practices and estimate the effect the pandemic on the anaesthesia providers as individuals. The overall objective was to learn from the collective experiences and establish a minimum benchmark to improve safety of both anaesthesia providers and patients and be better prepared should the pandemic continue, become more widespread, or, if there was to be another pandemic of similar nature.

\section{METHODS AND MATERIALS}

An anonymous 31-point questionnaire survey comprising of multiplechoice questions (MCQs) with option for comments (Appendix 1) was sent by email to 75 practicing ophthalmic anaesthesia providers from available contacts of attendees, speakers, and colleagues of the World Congress of Ophthalmic Anaesthesia (WCOA); British Ophthalmic Anaesthesia Society (BOAS); Ophthalmic Forum of Indian Society of Anaesthetists (OFISA); European Society of Ophthalmic Anaesthesia (ESOA) and some personal contacts of colleagues running nongovernment private units in different parts of world. The geographical distribution thus included Australia, Brazil, Egypt, France, Germany, India, Indonesia, Ireland, Italy, Russia, Singapore, South Africa, Switzerland, Turkey, UK and USA representing English and non-English speaking regions. There were no exclusion criteria as such except that we did not want to over represent any individual unit, or, region.

The questionnaire was ratified by the hospital Research \& Development Committee (Sandwell and West Birmingham Hospitals NHS Trust, Birmingham, UK) and, a formal ethics approval was deemed unnecessary. The survey was conducted electronically between December 2020 and January 2021. Blanket reminders via emails were sent in January/February 2021. Fifty-one responses were received giving a response rate of $68 \%$.

\section{Questionnaire design}

The questions were designed to assess the institutional and individual practices in delivering ophthalmic anaesthesia during Covid -19 pandemic. These questions included the structure of the ophthalmic units, services provided, practices, policies, and equipment for protection of staff and patients in relation to general anaesthesia (GA), regional/local anaesthesia (LA/RA), sedation/monitored anaesthesia care (MAC) during the strict lockdown and relaxation periods of the pandemic in respective countries of the respondents. We also tried to assess if respondents had felt if their individual personal protection in the eye units/hospitals was robust and fit for the purpose (or not), and if the pandemic had affected the way they administered ophthalmic LA/RA.

We wanted to know if there were any differences between the care provided to patients receiving non ophthalmic RA (peripheral nerve/ regional blocks) compared to ophthalmic LA/RA during the pandemic. The survey also endeavoured to seek if ophthalmic anaesthesia providers were redeployed to provide services in the intensive therapy units, acute Covid wards, or, other areas. The respondents were asked if reshuffling or sudden change of their job plans and serious illness/ deaths of their colleagues caused by Covid-19 had had any detrimental psychological effects on them.

The questions were very detailed but they were not mandatory as it was felt that some questions might be too sensitive for some respondents. For similar reasons no attempt was made to identify the institutions or link the countries to the respondents.

\section{RESULTS}

Even though the onset of lockdown and relaxation periods of different countries varied in duration and intensity, the overall essential protective strategies for staff and patient management were expected to be similar. As there were no mandatory questions, the number of responses received therefore varied for each question. Full results are included in Appendix 2.
A. Institutions, Individuals, and their usual practice in Prepandemic period

Seventy-eight percent of the respondents were anaesthetists, $22 \%$ were ophthalmologists. All units provided comprehensive ophthalmic services. Nearly sixty percent of the units/hospitals were funded by the government and $40 \%$ had private funding. Majority of the units (64.6\%) were part of a main hospital complex but located in a standalone building, $35.4 \%$ were totally standalone, and, independent.

Teaching and training units constituted $85.7 \%$; and $14.3 \%$ were non-teaching service units. Most units (75.5\%) served all ages (adult and paediatric), 16.3\% served adults and paediatric patients of age 3 years and over, $8.2 \%$ served only adults, no paediatrics.

$71.4 \%$ units provided all services (emergency and elective) on $24 / 7$ basis, $16.3 \%$ provided these during daytime only, and $12.2 \%$ delivered just elective work during daytime.

Patients of mixed races were served by $56.3 \%$ of the units, $22.9 \%$ served Caucasians/Europeans and 20.9\% served Southeast Asian/Indian population.

The majority (89.4\%) of the units had facilities to provide GA, $95.9 \%$ provide RA/LA of which $8.7 \%$ also provided sedation (MAC), and $85.7 \%$ units used topical of which only $7 \%$ offered sedation.

A vast majority of the respondents $(85.1 \%)$ were regular ophthalmic anaesthesia providers for both elective and emergencies, $14.9 \%$ only provided emergency on-call services. The usual practice of $37.5 \%$ respondents included both needle based extraconal (peribulbar) and sub-Tenon's blocks; extraconal blocks were used by $35.3 \%$, only subTenon's by $29.2 \%$, intraconal (retrobulbar) blocks by $4.45 \%$, topical by $4.2 \%$, sub- conjunctival injection by $2.1 \%$ and $4.2 \%$ did not do RA, just GA. $4.2 \%$ practiced none of these, infiltration LA only was done by $2.1 \%$.

B. Changes in service provision at the onset of Covid-19 pandemic compared to Pre- pandemic period

At the onset of the pandemic ophthalmic surgical services had been reduced in the units of $55.1 \%$ respondents, $8.2 \%$ had significant reduction, and $34.7 \%$ had no change, $2 \%$ respondents didn't know if a change had happened.

During the total lockdown, $54.9 \%$ responses indicated that all elective ophthalmic surgery had stopped, 38.8\% units had even further reduction in elective work, urgent/emergencies urgent cases continued as usual. $6.1 \%$ responded that there was no change from pre-pandemic or non- lockdown period, and $2 \%$ didn't know the answer.

C. Pre-admission Covid-19 antigen testing

C1. Elective General Anaesthesia (GA)

All elective GA patients in the respective units of $79.5 \%$ respondents were tested for Covid-19 antigen before admission, $12.2 \%$ respondents said only some patients were tested, $8.2 \%$ respondents' units conducted no test at all. Reasons for not testing included hospital policy (25.8\%), lack of time (19.4\%) and lack of testing kits (16.1\%).

C2. Urgent General Anaesthesia

For urgent GA cases, all patients in $60.5 \%$ respondents' units were tested, $25 \%$ units were testing only some patients, $12.6 \%$ conducted no tests, and $4.2 \%$ respondents didn't know if any tests were being done. Reasons for not testing emergency GA patients were hospital policy (23.7\%), lack of time (34.2\%) lack of testing kits (13.1\%), $2.6 \%$ didn't know the answer, others answers were not applicable.

C3. Elective Regional/Local anaesthesia (RA/LA) without sedation

in $44.9 \%$ of respondents' units all above patients received mandatory pre-admission Covid-19 antigen tests, whilst in $20.4 \%$ respondents' units only a few patients were tested, in 
$34.7 \%$ respondents' units these patients received no test at all. This (no tests) number is much higher when compared to elective GA patients (8.2\%). Reasons for not testing elective LA/RA (without sedation) patients were blanket hospital policy in $33.3 \%$ (note this number is higher than for elective GA which was $25.8 \%$ ). $25.6 \%$ respondents' units considered LA/RA patients not risky enough to warrant tests, $15.1 \%$ responses cited lack of testing kits etc, $5.4 \%$ mentioned lack of time and $2.6 \%$ lack of money, patient refusal accounted for $2.6 \%$, other responses were not applicable.

C4. Elective RA/LA with sedation/monitored anaesthesia care (MAC)

As per $53.2 \%$ responses, all patients receiving LA/RA + sedation/MAC had pre-admission covid testing (slightly higher than LA/RA without sedation), 14.9\% responses indicated that only some patients were tested, and $31.9 \%$ respondents said their units did no tests for these patients. The reasons were: blanket hospital policy not to test: $28.9 \%$; $23.7 \%$ respondents indicated that their units didn't consider LA/RA patients for sedation any riskier than LA/RA alone therefore did not justify testing; $13.2 \%$ cited lack of testing kits; $2.6 \%$ lack of time; and $2.6 \%$ lack of money. Remaining responses were not applicable.

D. Management of Non ophthalmic RA Vs Ophthalmic RA

As per $13 \%$ responses, the non-ophthalmic RA patients received different preoperative screening and testing procedures compared to ophthalmic ones, $69.6 \%$ respondents indicated that there was no difference, and $17.4 \%$ did not know the answer.

E. Peri-operative isolation, segregation and streaming of UNKNOWN, UNTESTED patients from COVID NEGATIVE patients

One third respondents (28.6\%) indicated that their units were not segregating their Covid-19 negative patients from those with unknown untested status patients, whilst $61.2 \%$ did, and $10.2 \%$ didn't know the answer to the question.

F. Provision of personal protective equipment (PPE) for staff conducting RA/LA for UNKNOWN/UNTESTED patients

Interestingly only $44.8 \%$ anaesthesia providers were provided full advanced level 3 PPE (Fluid repellent gowns, gloves, Silicon or FFP 3 masks, face shields, eye and hair protection etc) whilst $36.4 \%$ had patchy inconsistent PPE such as varying combinations of simple surgical masks, gloves and eye protection. $16.3 \%$ respondents wore just simple surgical mask and gloves but no gown or eye protection. $4 \%$ did not deal with untested covid status patients.

G. Other protective barriers during LA/RA

Patients wore simple face covering during LA/RA in 46\% respondents' units, $31.2 \%$ said that in a barrier drape was placed on face and torso of patients in addition to face covering, $6.3 \%$ used a hard plastic aerosol box to cover head and torso, $2.1 \%$ respondents used no additional barrier at all, 2.1\% left it to attending doctor, 2.1 didn't know the answer. Others (10.5\%) served only covid negative patients.

H. Redeployment of ophthalmic anaesthesia providers

Nearly $18.8 \%$ respondents were redeployed to acute Covid wards/ITU, $27.1 \%$ had reduced ophthalmic duty hours but were not redeployed, and $54.2 \% \%$ ophthalmic anaesthesia providers had no changes to their ophthalmic duties.

I. Regular Covid-19 antigen screening tests for patient facing staff

Patient facing staff were not being tested as per $65.3 \%$ responses, only $34.7 \%$ responses indicated that regular antigen tests for staff were being done in their units.
J. Life-threatening illness or death of a colleague within department due to confirmed Covid-19

Life-threatening serious illness or death of a colleague due to confirmed Covid-19 within the department was acknowledged by $16.3 \%$ of respondents, $81.6 \%$ had not come across this type of tragedy, and $2.1 \%$ did not know if there had been such issues in their units.

K. Concerns about personal health and well-being whilst serving patients with unknown, untested Covid-19 status

With respect to health and safety, 29.2\% respondents were very concerned about their personal well-being, $50 \%$ were somewhat concerned, only $20.8 \%$ were not concerned.

L. Impression about suitability and adequacy of PPE provision by the parent ophthalmic unit

Two thirds of the respondent (75\%) felt that PPE provision was adequate and safe, whilst $20.8 \%$ said it could have been better, $2.1 \%$ had access to better masks if required, $2.1 \%$ didn't work in high-risk area.

\section{DISCUSSION}

The results of the survey demonstrate that the respondents were not a homogenous group, and, their ophthalmic units were not equal. These differences withstanding, the virus doesn't make any distinction between a large or small, private or government funded unit, therefore, institutional/individual duty of care to patients and staff was expected to be similar.

At the onset of the pandemic, in general, most health care institutions were initially rather slow to protect the frontline workers, but later implemented policies to focus mainly on the emergency departments, and intensive care units. New policies included enhanced infection control in operating theatre; improved PPE for patient facing staff; screening patients for travel, contacts, systemic symptoms; mandatory pre-admission quarantine; pre-admission antigen tests for SARS-COV-2; and, on admission streaming patients into high, medium, and low risk depending on above criteria.

Our international survey of ophthalmic unit practices has identified that apart from cutting back of the elective operating services, preparedness and reaction to the pandemic, and, the methods employed to protect staff and patients were rather capricious, and at times, sub-optimal across the board. Ophthalmic anaesthetic units lagged behind their non-ophthalmic counterparts. Personal communication (SV) suggested that the practice to protect the patient facing Health Care Workers (HCWs) and anaesthesia providers/ophthalmologist varied widely not only from one hospital to another, but also across different departments within the same hospital.

It is well recognised that patient facing HCWs have been at an increased risk of contracting the virus during their daily work given the virulence and novelty of the SARS-COV-2. HCWs represented between $3.8 \%$ and $20 \%$ of the infected population in the early part of the pandemic [8]. According to published newspaper reports in the United Kingdom; 52000 NHS workers in the UK had been off sick with Covid-19, and over a six-month period (March-December 2020) there were 850 deaths amongst HCWs and social care workers [9]. In the USA deaths of HCWs had been reported to be around 3000 [10]. At the time of survey, sixteen ophthalmologists worldwide had been known to succumb to Covid -19 (American Academy). Re-current waves in some parts of the world such as India had resulted in countless further deaths of doctors and others HCWs.

The scientific literature and the World Health Organisation (WHO) had identified the high virulence of the contagion early on in the pandemic. Guidelines had been issued to identify and isolate suspected carriers [8]. There was plenty of evidence that not all carriers had overt symptoms, but were capable of 
onwards transmission. The only way to know if the person was a carrier was to perform a formal test for the contagion. Unfortunately, in this survey, pre-operative patient testing for antigen (RT-qPCR) wasn't seen to be a requirement especially for RA/LA patients. This was true for even elective GA patients for whom in $(8.5 \%)$ units no tests were conducted at all. Hospital policy, sadly, was responsible for not conducting pre-operative Covid antigen tests as per 1/4th of the responses; lack of testing kits and lack of time constituted other reasons, but it is not understandable why, and, how there wasn't enough time for testing Elective patients.

As far as urgent GA cases were concerned, $37.6 \%$ units not conducting tests is possibly understandable as in the initial phases of the pandemic the antigen test (RT-qPCR) had slow turn around, yet some units $(60.5 \%)$ performed better than others. Lack of time (34.2\%) as a cause for not testing is a reasonable explanation but why a quarter of ophthalmic units implemented blanket policies not to test urgent GA patients at all, is not comprehensible.

Not testing Elective RA/LA patients is even more baffling. No pre-admission tests were done in $31.9 \%$ units (LA/RA + sedation), and in $34.7 \%$ (no sedation) group compared with $8.2 \%$ in elective GA set. Inconsistent/random testing was done in $14.9 \%$ (LA/RA + sedation) and $20.4 \%$ (LA/RA, no sedation) versus $12.4 \%$ for elective GA.

It is noteworthy that the volume of RA/LA had increased significantly during the pandemic to minimise general anaesthetics. The UK was an example where such transition had taken place and other countries wouldn't have been any different [11]. Maintenance of social distance during administration of RA/LA is not practical. The fact that $1 / 5^{\text {th }}$ of ophthalmic units (irrespective of RA or GA) didn't even formally segregate untested unknown covid patients from those who were covid negative, is alarming.

Furthermore, the fact that patients for non-ophthalmic RA had better/different perioperative screening/testing procedure compared to their ophthalmic counterparts within same hospital setting was even more puzzling.

It is possible that the reasons for above was the way ophthalmic RA had been perceived (incorrectly) in the context of aerosol transmission of this contagion. The initial focus had solely been on the AGPs (Aerosol generating hospital procedures, e.g. GA, intermittent positive pressure ventilation, intubation, extubation, suction, coughing etc) as opposed to AGBs (aerosol generating behaviours) e.g. breathing, speaking, loud phonation, sneezing, coughing, singing etc). Whilst it is well established that AGPs in infected patient produce high viral loads, AGBs also generate substantial droplets and aerosols, therefore their role in contagion transmission from an infected but undiagnosed patient must not be underestimated [12-22].

Ophthalmic RA awake patients tend to cough (often incessantly due to COPD), speak, (often loudly), and may sneeze during theatre journey. It is therefore conceivable that viral spillage and risk is no less than GA, and may in fact be more from untested, unsegregated patient especially during prolonged RA procedures (e.g. complex Cataract, Vitreoretinal or Glaucoma). During GA, the patient's airway is sealed (Laryngeal mask airway/Endotracheal tube), unlike in RA. Additionally, the guard is down during RA/LA whereas in GA staff are more cautious, better equipped, and properly protected.

The dispersion of virus laden droplets/aerosols is dependent on the particle size. Large droplets $(>5 \mu \mathrm{m})$ generally migrate to about $<2 \mathrm{~m}$ and may fall downwards due to gravity, while smaller lighter aerosols float about for much longer and may disperse as far as $8 \mathrm{~m}$ [23]. When inhaled, the larger particles $(>5-10 \mu \mathrm{m})$ deposit in the upper airway, medium-sized (1-5 $\mu \mathrm{m})$ travel to smaller airways and those measuring $<1 \mu \mathrm{m}$ are able to infiltrate even respiratory alveoli [24].

The range these droplets/aerosols disperse is dependent on the ambient relative humidity $(\mathrm{RH})$ and air-currents. $\mathrm{RH}$ of $40 \%-60 \%$ tends to limit the dispersion. A low $\mathrm{RH}$ of $<40 \%$ dries out droplets enabling the virus to travel out much further out and remain viable for longer [25]. The virus can remain viable in aerosols for 3 h [26]. SARS-CoV-2 has also been isolated from hospital air [27, 28]. Covid-19 positive patients have been shown to emit billions of SARS- CoV-2 RNA particles into air [29], In hospitals, whilst operating theatres have reasonably well-regulated $\mathrm{RH}$ and air flows; recovery areas, congested consultation rooms/wards do not have same facilities. It therefore entirely likely that units which did not test/stream their patients or staff, these dense spaces would have carried a high ambient viral load risking HCWs.

Sedation and supplemental nasal oxygen administration in undiagnosed/asymptomatic carriers receiving ophthalmic RA is a further cause for concern when it comes to viral dispersion. The fact that a significant number of ophthalmic units didn't perceive pre-operative testing even for sedated RA/LA patients, is very worrying.

PPE along with other measures, is one way of avoiding contracting the virus, but it needs to be fit for the purpose. In a high-risk red/amber area, all exposed body surfaces need to be protected by level 3 PPE to prevent inhalation/ingestion of virus particles. This is usually achieved by combination of individually fitted Silicon, FFP3 or N95 face masks, fluid repellent gowns, eye protection, face shields, gloves, hair covers; and a formal training in correct donning and doffing [30-32]. In this survey several respondents felt that their overall PPE was not as good as could have been. Over half of the ophthalmic anaesthesia providers didn't have the enhanced level 3 PPE; and $40 \%$ had only basic kits. With respect to RA, whilst some practitioners did have level $3 \mathrm{PPE}$, many just had minimum basic items e.g., simple surgical face mask and gloves. The notion that ophthalmic RA/LA patients do not pose a risk of transmission to HCWs, clearly represents a lack of understanding of viral dispersion. Above notion, interestingly was also revealed in a study which showed a low perceived confidence, a deficit in knowledge and education amongst U.K. doctors across all specialities when it came to PPE [33]. As it happens, in this study, ophthalmology had the lowest scores in the knowledge based multiple choice questions.

The survey showed that the usage of additional active barrier between patients and anaesthesia provider varied significantly during administration of RA. A large majority of respondents relied on patients wearing a simple face covering. These poorly fitted, contaminated face coverings, which get pulled up and down during patients' hospital journey are preordained to fail. Some practitioners did use fluid repellent adhesive drapes or a novel plastic box [34] to cover these masks, they were exception rather than norm. Viral RNA has been reported in the ocular-surface samples and conjunctival exudate $[35,36]$. Ocular surface viruses can be inactivated by careful pre-block disinfection with povidone iodine, there is no guarantee of their clearance from the surrounding skin, hence onward transmission remains a real risk.

As regards screening of patient facing staff, even as late as January 2021, in $65.3 \%$ of ophthalmic units, staff were not being screened for SARS-Cov-2 antigen. This was once again more of an institutional decision rather than an individual one. Reliable RtqPCR tests had been available since the first lockdown, self-test kits (rapid lateral flow) were obtainable for several months before the survey, but it appears that testing for patient facing staff was amiss amongst other things.

The pandemic has had a definitive impact on personal physical and psychological wellbeing of HCWs [37]. Severe staffing difficulties were experienced by most institutions. Resources were redirected to ITUs caring for Covid-19 patients as elective procedures came to a halt [11]. HCWs were redeployment to acute units where an alien environment, unfamiliar equipment and protocols, complex intensive care interventions, apprehension about medicolegal adversities, personal health and safety, all took their toll. There was scant induction or re-training for these new 
roles hence most people found 'on the job' learning curve incredibly stressful and very steep, (personal communication). Although some targeted literature was available for staff [38], it was not sufficient to allay anxieties. In this survey $18.8 \%$ respondents were redeployed to acute Covid units. Whilst ophthalmic anaesthetists performed at the deep end of critical care, other staff (including ophthalmologists), were deployed for 'proning' of Covid patients, maintenance of surface sterility, and porter services. Some respondents had witnessed Covid related severe illness and death amongst own colleagues/department. A large number said that they were concerned about their personal safety whilst serving patients with unknown covid status.

\section{CONCLUSION}

The survey is first of its kind in highlighting the impact of Covid19 pandemic relating to ophthalmic anaesthesia providers. The results have shown that ophthalmic services worldwide had inadequate attention to the reality and threat of the pandemic. Preoperative screening of patients and streaming was inconsistent for GA, and inadequate for RA/LA. Provision of PPE for staff during RA/LA was not optimal. Even general anaesthesia set up was lack-lustre. The redeployment to unfamiliar acute units and severity of the pandemic had had an adverse psychological impact on the staff.

Ophthalmic HCWs are a valuable and irreplaceable resource. We hope that the results will help in the preparedness for continuing waves and future pandemic of similar magnitude. It is imperative that going forwards robust, mandatory, preventive measures and practices are put in place to avoid potential physical and psychological damage.

\section{Limitations of the survey}

Authors acknowledge that this survey is not without limitations. A possible selection biases exists as anaesthesia providers in different countries follow their own local norms and protocols. In order to conduct the survey, we required ophthalmic anaesthesia providers serving actively in different parts of the world. Since we were not aware of any international database of such providers, we had approached Ophthalmic Anaesthesia Societies (they are limited to India, the UK and USA) with the hope of using their databases for recruitment of the participants, but unfortunately, we were denied access due to prevailing data protection acts.

The only option available to us was to use the contacts of organisers and delegates of the past ophthalmic anaesthesia conferences with a hope that they were still engaged in providing ophthalmic anaesthesia at the time of this survey. The recruitment was not done during any active conference as such, hence there was no personal influence, or peer pressure. Moreover, the responders were under no obligation to participate, or answer every question.

In terms of the questions, certain aspects of clinical practice were deemed to be too sensitive for the respondents and their institutions. Fear of medico-political trepidations meant that the questions relating to any loss of income (e.g., locums) when the elective ophthalmic work ceased, or the psychological impact of prolonged mandatory shielding/isolation for those who were not allowed to work due to institutional health and safety directives, were not asked.

We intentionally did not attempt to quantify the actual transmission of contagion within a given unit as it wasn't the aim of the survey. There were already enough details of morbidity/ mortality published in the world media. We just wanted to see if the units had attentiveness to prevent virus from spreading; and if effective practices were in place to protect their precious workforce and patients from avoidable harm.

\section{Summary}

What was known before

- There has never been a pandemic like Covid-19 before.

- There is no previous literature available that specifically highlights the effect of a pandemic like covid-19 on the ophthalmology services and its anaesthesia services providers worldwide.

What this study adds

- This survey is first of its kind studying the impact Covid-19 pandemic relating to ophthalmic services and its anaesthesia providers.

- The results revealed that ophthalmology and ophthalmic anaesthesia service worldwide had inadequate attention to the reality and threat of the pandemic Preoperative patient screening, segregation, covid-19 antigen testing particularly for regional anaesthesia patients and PPE was sub-optimal. Ophthalmic Staff were redeployed to ITU and acute wards, eye services were cut back, and staff screening for the antigen was sporadic.

- The lack of effective screening processes, and protective measures for staff, even for general anaesthesia, bordered on being unsafe.

- It is imperative that going forwards robust, mandatory, preventive measures and practices are put in place to avoid irreversible physical and psychological disorders in Ophthalmic healthcare workforce.

- Authors recommended that institutional and individual practices are modified by training, education, and cross fertilisation of ideas.

- Procedures and policies based on scientific evidence need to be standardised with the aim to maintaining a goal of zero transmission of the current, or a future contagion.

- The patients receiving regional ophthalmic anaesthesia are just as capable of onwards transmission of the contagion due to $A G B s$, as are their general anaesthesia counterparts undergoing AGPs.

- Given that the pandemic is continuing to rage in some parts of the world despite vaccination, we sincerely believe there is no room for complacency.

\section{REFERENCES}

1. Coronavirus kills Chinese whistle blower ophthalmologist. American Academy of Ophthalmology. 2020 [cited 2021 May 4]. https://www.aao.org/headline/ coronavirus-kills-chinese-whistleblower-ophthalmol.

2. Peiris JSM, Yuen KY, Osterhaus ADME, Stöhr K. The severe acute respiratory syndrome. N Engl J Med. 2003;349:2431-41. Dec 18

3. Raboud J, Shigayeva A, McGeer A, Bontovics E, Chapman M, Gravel D, et al. Risk factors for SARS transmission from patients requiring intubation: a multicentre investigation in Toronto, Canada. PLoS ONE. 2010;5:e10717. May 19

4. Tong T, Lai TS. The severe acute respiratory syndrome coronavirus in tears. $\mathrm{Br} \mathrm{J}$ Ophthalmol. 2005;89:392. Mar

5. Loffredo L, Pacella F, Pacella E, Tiscione G, Oliva A, Violi F. Conjunctivitis and COVID-19: A meta-analysis. J Med Virol. 2020;92:1413-4. Sep

6. Jevnikar K, Jaki Mekjavic P, Vidovic Valentincic N, Petrovski G, Globocnik Petrovic M. An update on COVID-19 related ophthalmic manifestations. Ocul Immunol Inflamm. 2021;29:684-9.

7. Baig AM, Ahmad S, Khaleeq A, Rafiqu H, Rajput S, Angez M, et al. Ocular COVID19: Eyes as a reservoir to conceal and spread SARS-CoV-2. Infect Disord Drug Targets. 2020; https://doi.org/10.2174/1871526520999200729182242.

8. Kowalski LP, Sanabria A, Ridge JA, Ng WT, de Bree R, Rinaldo A, et al. COVID-19 pandemic: effects and evidence-based recommendations for otolaryngology and head and neck surgery practice. Head Neck. 2020;42:1259-67. Jun 
9. More than 850 health and social care workers have died of Covid in England and Wales since the pandemic began [Internet]. [cited 2021 Apr 11]. https://www. yorkshireeveningpost.co.uk/health/coronavirus/more-850-health-and-social-careworkers-have-died-covid-england-and-wales-pandemic-began-3114202.

10. Our key findings about US healthcare worker deaths in the pandemic's first year. The Guardian [Internet]. [cited 2021 Apr 11]; Available from: https://www.theguardian. com/us-news/ng-interactive/2020/dec/22/lost-on-the-frontline-our-findings-to-date.

11. RCO: RCOphth: Management of Ophthalmology Services during the Covid pandemic [Internet]. The International Agency for the Prevention of Blindness. https://www.iapb. org/learn/resources/rco-rcophth-management-of-ophthalmology-services-during-thecovid-pandemic/.

12. Balachandar S, Zaleski S, Soldati A, Ahmadi G, Bourouiba L. Host-to-host airborne transmission as a multiphase flow problem for science-based social distance guidelines. Int J Multiph Flow. 2020;132:103439. https://www.sciencedirect.com/ science/article/pii/S0301932220305498.

13. Morawska L, Johnson GR, Ristovski ZD, Hargreaves M, Mengersen K, Corbett S, et al. Size distribution and sites of origin of droplets expelled from the human respiratory tract during expiratory activities. J Aerosol Sci [Internet]. 2009;40:256-69. https:// www.sciencedirect.com/science/article/pii/S0021850208002036.

14. Netz RR. Mechanisms of airborne infection via evaporating and sedimenting droplets produced by speaking. J Phys Chem B. 2020;124:7093-101. https://doi. org/10.1021/acs.jpcb.0c05229.

15. Yang S, Lee GWM, Chen C-M, Wu C-C, Yu K-P. The size and concentration of droplets generated by coughing in human subjects. J Aerosol Med. 2007;20:484-94.

16. Stelzer-Braid S, Oliver BG, Blazey AJ, Argent E, Newsome TP, Rawlinson WD, et al. Exhalation of respiratory viruses by breathing, coughing, and talking. J Med Virol 2009;81:1674-79. Sep

17. Asadi S, Wexler AS, Cappa CD, Barreda S, Bouvier NM, Ristenpart WD. Aerosol emission and super-emission during human speech increase with voice loudness. Sci Rep. 2019;9:2348. https://doi.org/10.1038/s41598-019-38808-z.

18. Lindsley WG, Pearce TA, Hudnall JB, Davis KA, Davis SM, Fisher MA, et al. Quantity and size distribution of cough-generated aerosol particles produced by influenza patients during and after illness. J Occup Environ Hyg. 2012;9:443-49.

19. Tang JW, Nicolle AD, Klettner CA, Pantelic J, Wang L, Suhaimi AB, et al. Airflow dynamics of human jets: sneezing and breathing - potential sources of infectious aerosols. PLoS ONE. 2013;8:e59970.

20. Tang JW, Nicolle A, Pantelic J, Koh GC, Wang LD, Amin M, et al. Airflow dynamics of coughing in healthy human volunteers by shadowgraph imaging: an aid to aerosol infection control. PLoS ONE. 2012;7:e34818.

21. Hirota K. Air contamination with SARS-CoV-2 in the operating room. J Anesth. 2021;35:333-36.

22. Morawska L, Cao J. Airborne transmission of SARS-CoV-2: The world should face the reality. Environ Int. 2020;139:105730.

23. Bourouiba L. Turbulent gas clouds and respiratory pathogen emissions: potential implications for reducing transmission of COVID-19. JAMA 2020;323:1837-38.

24. Zuo YY, Uspal WE, Wei T. Airborne transmission of COVID-19: aerosol dispersion, lung deposition, and virus-receptor interactions. ACS nano. 2020;14:16502-24.

25. Chong KL, Ng CS, Hori N, Yang R, Verzicco R, Lohse D. Extended lifetime of respiratory droplets in a turbulent vapor puff and its implications on airborne disease transmission. Phys Rev Lett. 2021;126:034502.

26. van Doremalen N, Bushmaker T, Morris DH, Holbrook MG, Gamble A, Williamson $\mathrm{BN}$, et al. Aerosol and surface stability of SARS-CoV-2 as compared with SARSCoV-1. N Engl J Med. 2020;382:1564-67. Apr 16

27. Guo Z-D, Wang Z-Y, Zhang S-F, Li X, Li L, Li C, et al. Aerosol and surface distribution of severe acute respiratory syndrome coronavirus 2 in hospital wards, Wuhan, China, 2020. Emerg Infect Dis. 2020;26:1583-91. Jul

28. Liu Y, Ning Z, Chen Y, Guo M, Liu Y, Gali NK, et al. Aerodynamic analysis of SARSCoV-2 in two Wuhan hospitals. Nature 2020;582:557-60.

29. Ma J, Qi X, Chen H, Li X, Zhang Z, Wang H, et al. COVID-19 patients in earlier stages exhaled millions of SARS-CoV-2 per hour. Clin Infect Dis. 2020; https://doi. org/10.1093/cid/ciaa/1283.
30. Karlsson U, Fraenkel C-J. Complete protection from covid-19 is possible for health workers. BMJ. 2020:370:m2641 https://www.bmj.com/content/370/bmj. $\mathrm{m} 2641$.

31. Koh FH, Tan MG, Chew M-H. The fight against COVID-19: disinfection protocol and turning over of CleanSpace ${ }^{\circledast} \mathrm{HALO}^{\mathrm{TM}}$ in a Singapore Hospital. Updates Surg 2020;72:311-13. Jun

32. Liu $M$, Cheng S-Z, Xu K-W, Yang $Y$, Zhu Q-T, Zhang $H$, et al. Use of personal protective equipment against coronavirus disease 2019 by healthcare professionals in Wuhan, China: cross sectional study. BMJ 2020;369:m2195.

33. Al-Hity S, Bhamra N, Kumar R, Gupta KK, Howard J, Jolly K, et al. Personal protective equipment guidance during a global pandemic: a statistical analysis of National perceived confidence, knowledge and educational deficits amongst UKbased doctors. Int J Clin Pract. 2021;75:e14029. May

34. Jaichandran VV, Raman R. Aerosol prevention box for regional anaesthesia for eye surgery in COVID times. Eye (Lond). 2020;34:2155-56. Dec

35. Cavalleri M, Brambati M, Starace V, Capone L, Nadin F, Pederzolli M, et al. Ocular features and associated systemic findings in SARS-CoV-2 Infection. Ocul Immunol Inflamm. 2020;28:916-21. Aug 17

36. Lauermann $P$, Storch $M$, Weig $M$, Tampe B, Winkler $M$, Hoerauf $H$, et al. There is no intraocular affection on a SARS-CoV-2 - Infected ocular surface. Am J Ophthalmol Case Rep. 2020;20:100884. Dec

37. Danet Danet A. Psychological impact of COVID-19 pandemic in Western frontline healthcare professionals. A systematic review. Med Clin (Barc). 2021;156:449-58. https://doi.org/10.1016/j.medcli.2020.11.009.

38. Harvey JP, Sinclair VF. Preparing ophthalmologists for the use of mechanical ventilation during the COVID-19 pandemic. Eye (Lond). 2020;34:1251-52.

\section{ACKNOWLEDGEMENTS}

We thank all the participating international ophthalmic anaesthesia providers (anaesthetists and ophthalmologists) for completing and returning the survey form during the pandemic.

\section{AUTHOR CONTRIBUTIONS}

SBV conceptualised and designed the questionnaire, obtained ratification of the questionnaire, and approval to conduct the survey (Hospital Research \& Development Committee, Midland Eye Hospital, Birmingham, UK), undertook survey administration, analysis of data, conducted literature search, drafted original manuscript and subsequent revisions. CMK conceptualised and designed survey questionnaire, conducted literature search, drafted original manuscript and subsequent revisions. All authors have read and approved the final manuscript.

\section{COMPETING INTERESTS}

The authors declare no competing interests.

\section{ADDITIONAL INFORMATION}

Supplementary information The online version contains supplementary material available at https://doi.org/10.1038/s41433-022-01979-7.

Correspondence and requests for materials should be addressed to Shashi B. Vohra.

Reprints and permission information is available at http://www.nature.com/ reprints

Publisher's note Springer Nature remains neutral with regard to jurisdictional claims in published maps and institutional affiliations. 